\title{
The utility of multidetector computed tomography for evaluation of congenital heart disease
}

\author{
U. Bayraktutan ${ }^{1}$, M. Kantarci ${ }^{1}$, H. Ogul ${ }^{1}$, Y. Kizrak ${ }^{1}$, B. Pirimoglu ${ }^{1}$, B. Genc ${ }^{2}$, \\ Z. Yuceler ${ }^{1}$, N. Ceviz ${ }^{3}$ \\ ${ }^{1}$ Department of Radiology, School of Medicine, Ataturk University, Erzurum, Turkey \\ ${ }^{2}$ Department of Radiology, School of Medicine, Sifa University, Izmir, Turkey \\ ${ }^{3}$ Department of Paediatric Cardiology, School of Medicine, Ataturk University, Erzurum, Turkey
}

[Received 4 April 2013; Accepted 4 May 2013]

Background: Congenital heart diseases (CHD) are the leading cause of birth defect-related deaths. Multidedector computed tomography (MDCT) plays an important role for imaging CHD in addition to echocardiography and provides a comprehensive evaluation of complex heart malformations for the referring cardiologist. The aim of the study was to evaluate the utility of MDCT in the assessment of $\mathrm{CHD}$.

Materials and methods: A 102 patients with CHD were investigated after initial assessment by echocardiography. The information obtained by MDCT and findings of echocardiography were reviewed together by paediatric cardiologists and cardiac radiologists. Perioperative anatomic descriptions, wherever available $(n=34)$ formed the gold standard for the comparison.

Results: The clinical consensus diagnosis defined 154 cardiovascular lesions in the patients. The results were classified in groups. We present the appearance of various congenital cardiac lesions seen in clinical practice.

Conclusions: MDCT provides important information about anatomic details of CHD for the referring cardiologist. The evaluation of different anatomic structures such as heart, great vessels, lungs and abdomen is possible in one acquisition with this technique. (Folia Morphol 2013; 72, 3: 188-196)

Key words: congenital heart disease, multidedector computed tomography

\section{INTRODUCTION}

Congenital heart disease (CHD) is one of the difficult issues in paediatric imaging because of the small size, complex cardiovascular morphology and rapid circulation. Cardiac imaging provides structural and functional information essential for treatment and follow-up complications after the treatment. Echocardiography is the initial imaging method for the diagnosis and management of CHD. This modality is operator dependent and limited by an acoustic window. Sometimes it is inadequate to show complex spatial relationships and provides incomplete visualisation of the extracardiac vasculature. Conventional angiography is usually used as the gold standard for diagnosing CHD, however, it has some disadvantages related to the invasive nature of the study, the need for general anaesthesia, and the enhanced sensitivity of neonates to radiation and iodinated contrast agent

Address for correspondence: M. Kantarci, MD, Department of Radiology, School of Medicine, Ataturk University, 200 Evler Mah. 14. Sok No 5, Dadaskent, Erzurum, Turkey, tel: +90 4423273802 (home), +90 442 2361212-1521 (work), fax: +90 (442) 2361301, e-mail: akkanrad@hotmail.com 

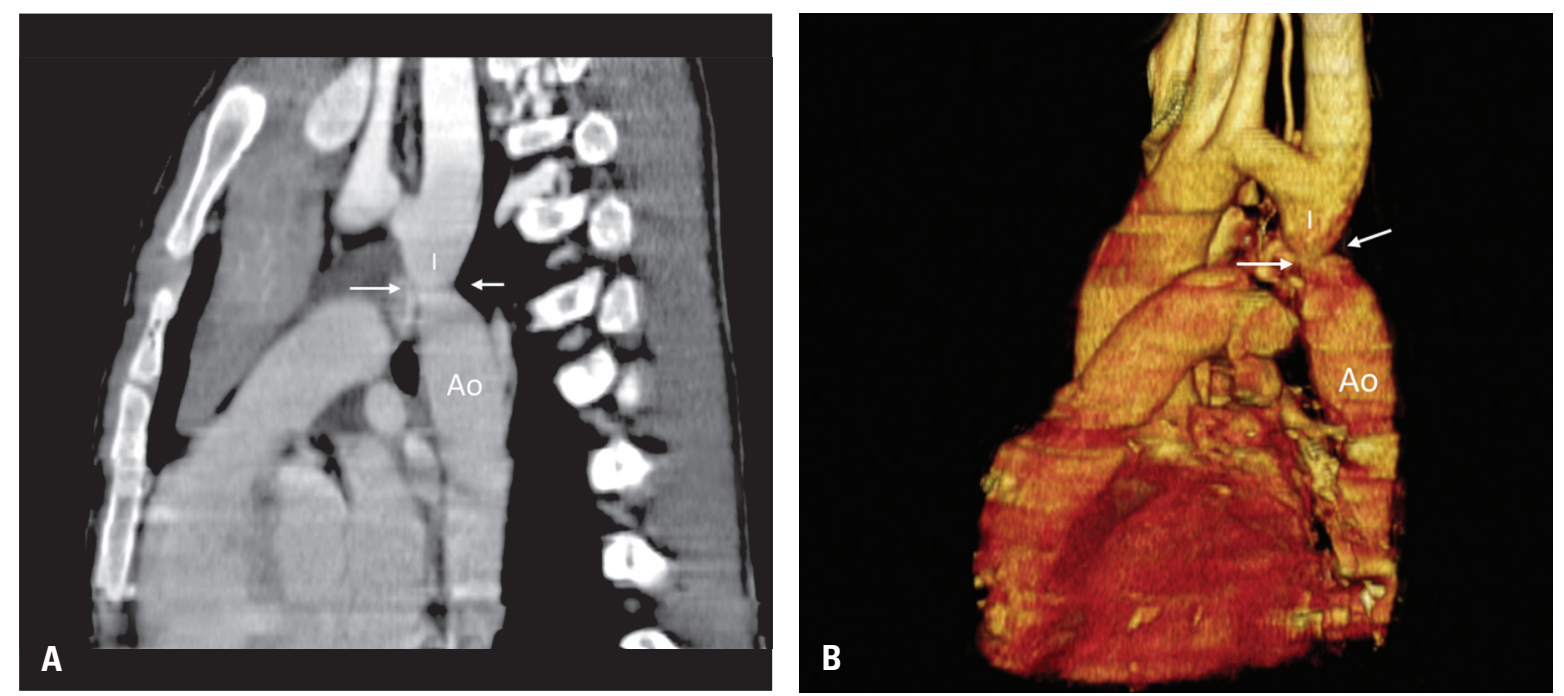

Figure 1. Sagittal maximum intensity projection image (A) and volume-rendered image (B) showing aortic coarctation; Ao — aorta; arrows - coarctation; I - isthmus.
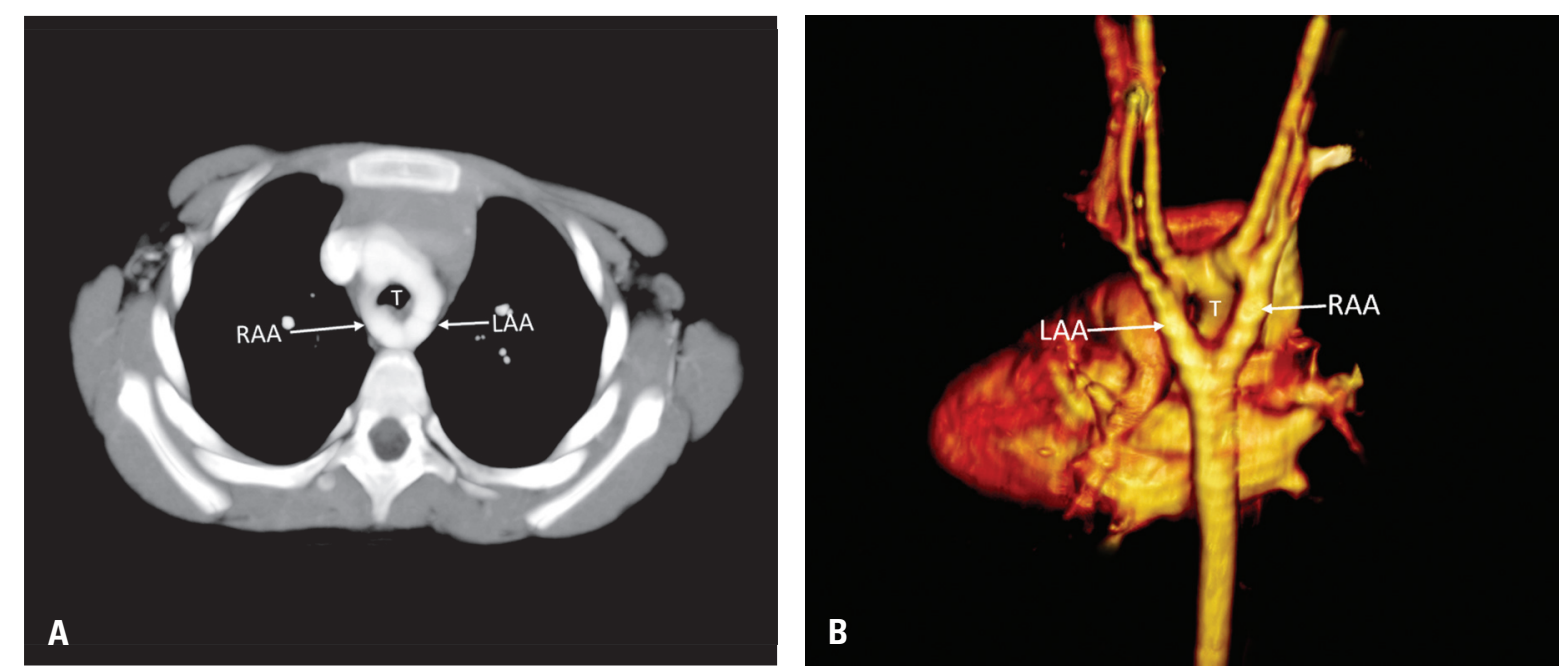

Figure 2. Axial maximum intensity projection image (A) and volume-rendered image (B) showing double aortic arch; LAA — left aortic arch; RAA — right aortic arch; T — trachea.

toxicity. After recent advances in computed tomography (CT) and magnetic resonance imaging (MRI) technologies, diagnostic cardiac catheterisation was no longer necessary.

Though MRI clearly shows the intracardiac anatomy, flow and function, clinicians' interest frequently focuses on the morphology of the extracardiac vasculature, including the coronaries, pulmonary arteries, aorta, and pulmonary or systemic veins. Multidedector computed tomography (MDCT) has the ability to show these structures with the vessel walls and also provides better delineation of the airway, mediastinal abnormalities, and the pulmo- nary parenchyma. MDCT is rapid, with a reduced need for sedation, is efficacious in the setting of metallic hardware, pacemakers and coils and is widely available, while MRI has limited availability, is time-consuming and requires sedation or general anesthesia. This study will focus on the utility of MDCT in the assessment of CHD.

\section{MATERIALS AND METHODS}

A total of 102 children and young adults (0-15 years; median 5.8 years) with CHD, after initial assessment by echocardiography between 2009 and 2012 were enrolled in this study. The medical ethics committee 

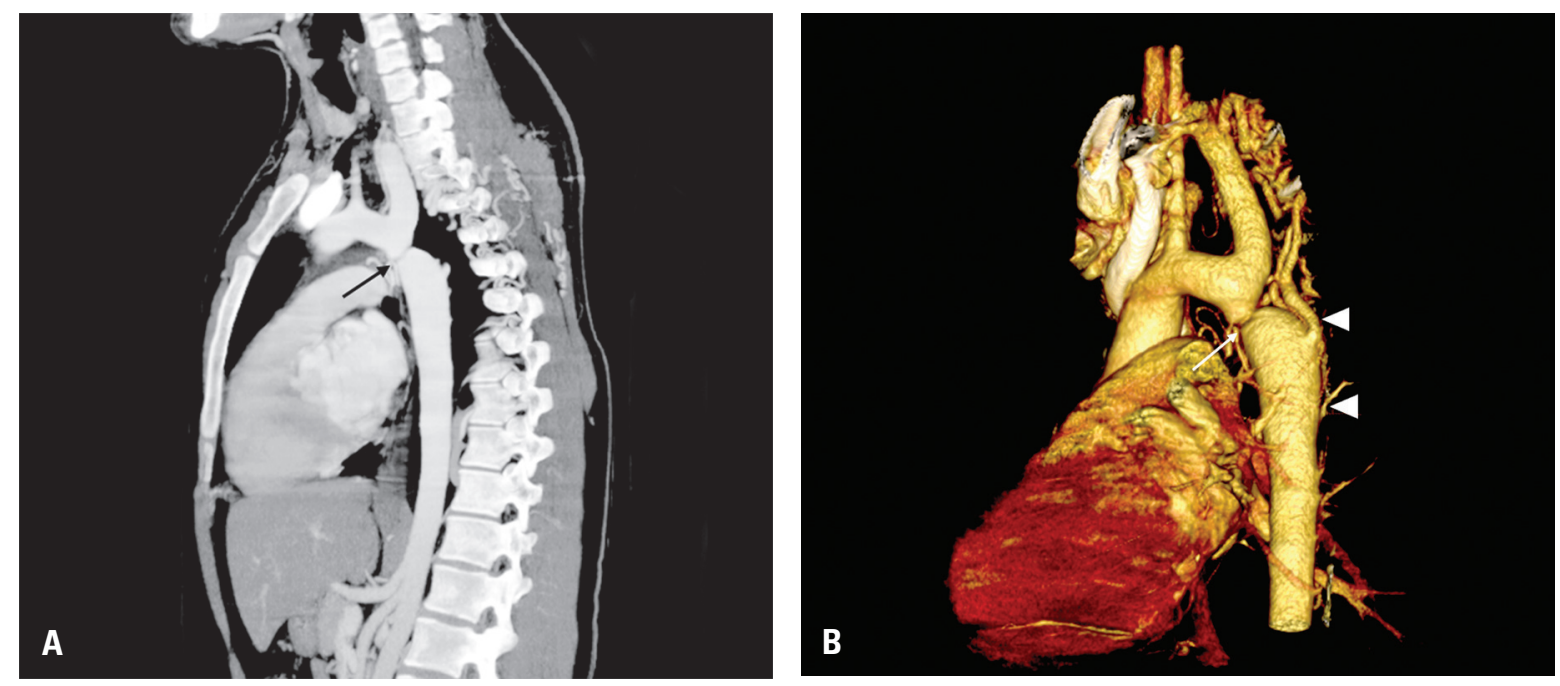

Figure 3. Sagittal maximum intensity projection image (A) and volume-rendered image (B) showing interrupted aortic arch; black arrow interruption of the arch (A); arrowheads - collaterals; white arrow — interruption of the arch (B).
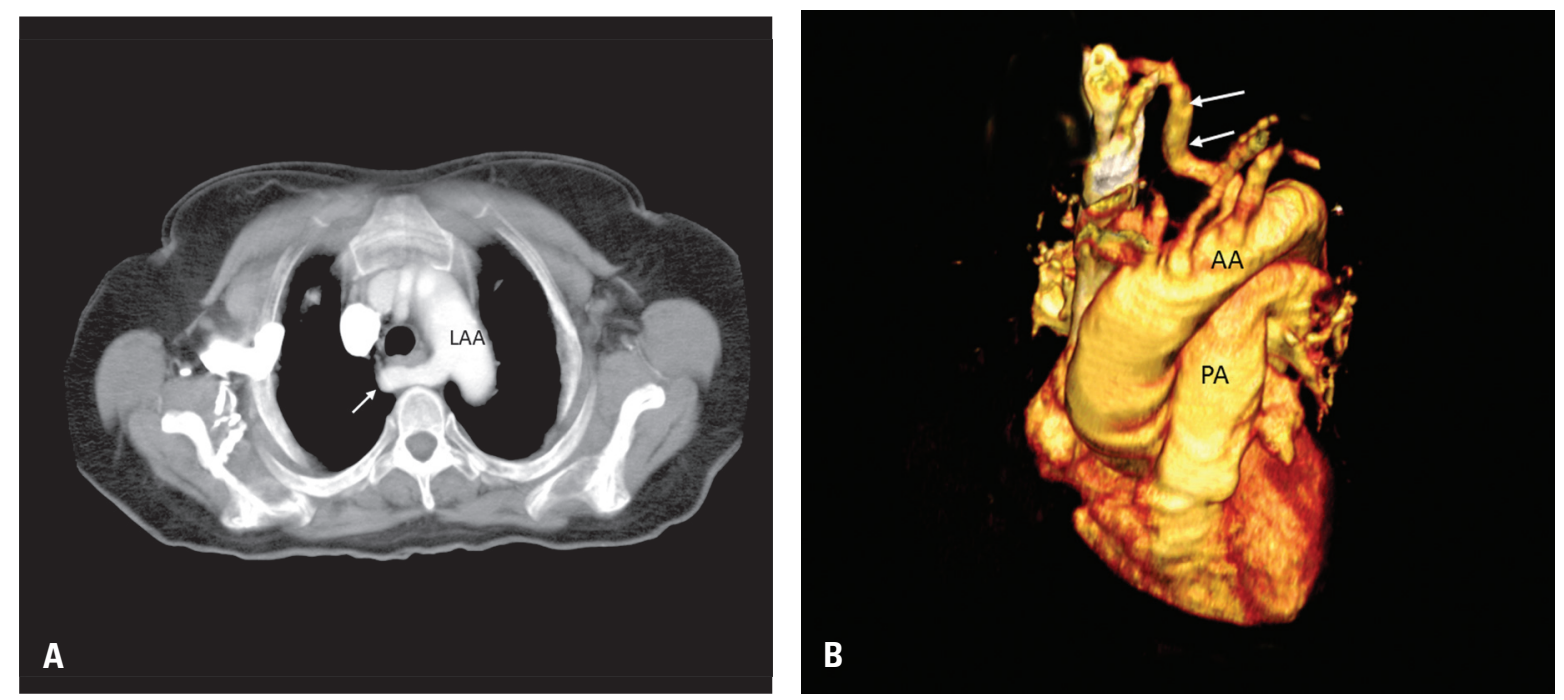

Figure 4. Axial maximum intensity projection image (A) and volume-rendered image (B) showing aberrant right subclavian artery; arrows aberrant right subclavian artery; LAA — left aortic arch; AA — aortic arch; PA — pulmonary artery.

of our hospital approved the study, and written consent of the patients and/or parents was obtained. Three patients with poor image quality due to motion artifacts were excluded. All the studies were performed in the presence of a qualified paediatric cardiologist and radiologist working in unison.

MDCT was performed with patients in the supine position using 256-slice helical CT (SOMATOM Definition Flash CT, Siemens Medical Solutions, Erlangen, Germany) and 16-slice CT scanner (Aquillion 16; Toshiba Corporation, Medical System Company, Tokyo, Japan). Sedation was achieved with either 50-100 mg/kg of oral chloral hydrate or 2-6 mg/kg of intravenous pentobarbital. MDCT scan parameters for the first scanner and the second scanner were respectively: pitch, 0.39 and 1.25; effective thickness, $0.9 \mathrm{~mm}$ and $1.0 \mathrm{~mm}$; voltage, $80 \mathrm{kV}$ and $120 \mathrm{kV}$; tube current, $120 \mathrm{~mA}$ and $120-300 \mathrm{~mA}$; rotation time, $0.28 \mathrm{~s}$ and $0.50 \mathrm{~s}$; scan time, 9-11 s and 4-16 s. The scan was from the upper margin of the shoulder to the lower margin of the liver. Electrocardiography and respiration-gated techniques were not used. No medication was used to lower or control the heart rate. Patient heart rates ranged between 60 and $150 \mathrm{bpm}$. Patients received $2.0 \mathrm{~mL} / \mathrm{kg}$ of contrast medium (Omnipaque, Amersham Health, Cork, Ireland) with a flow rate of $2-3 \mathrm{~mL} / \mathrm{s}$ 

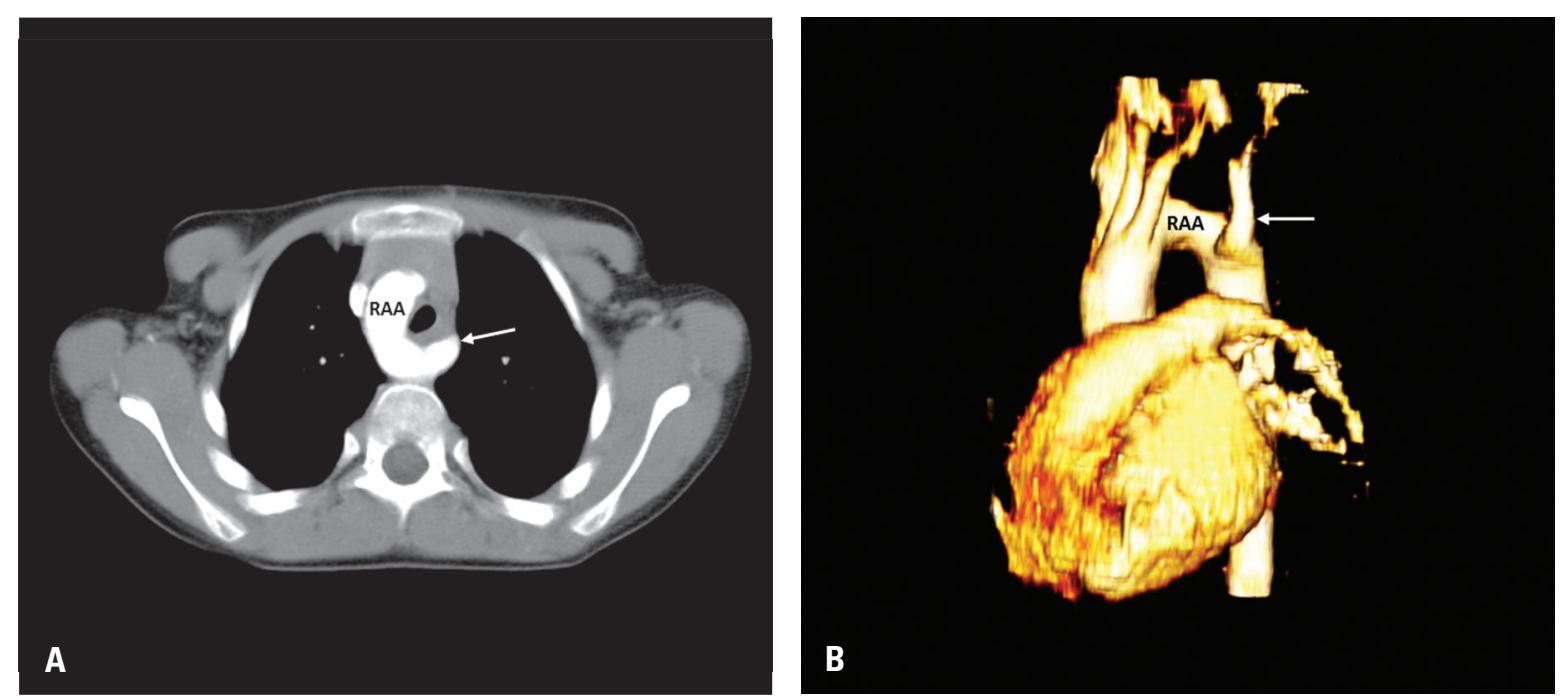

Figure 5. Axial maximum intensity projection image (A) and volume-rendered image (B) showing right aortic arch (RAA).
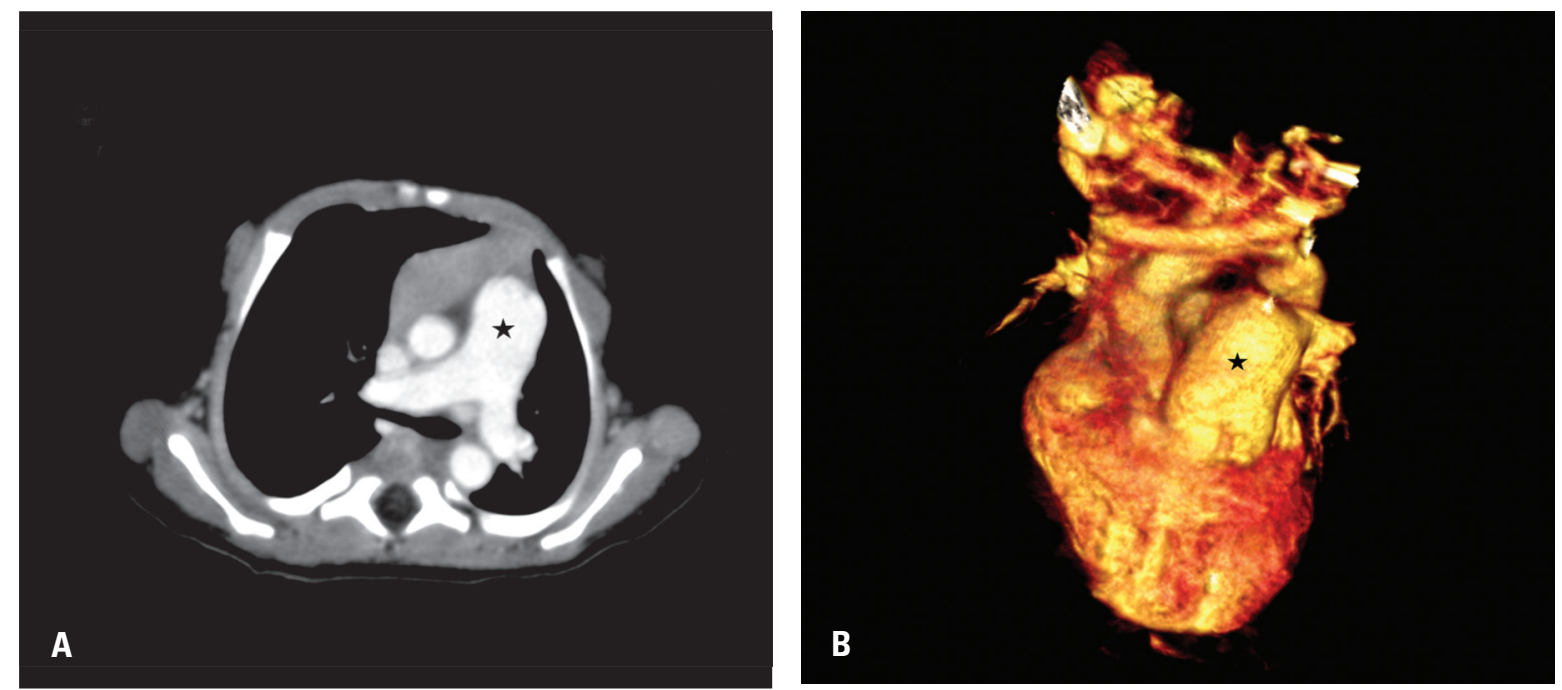

Figure 6. Axial image (A) and volume-rendered image (B) showing pulmonary artery dilatation in a patient with Marfan syndrome; star dilated pulmonary artery.

intravenously via an antecubital vein using a 22-gauge catheter. Images depicting a region of interest in the aorta or pulmonary artery were obtained with an acquisition delay of $4 \mathrm{~s}$ and an automatic triggering threshold of 100 Hounsfield Unit (HU).

All the source images were interpreted and postprocessed on two workstations (Syngo, Leonardo Workstation, Siemens and Vitrea 2, Vital Images, Minnetonka, MN, USA). Axial source, multiplanar reconstruction, maximum intensity projection, and volume-rendered images were used for the assessment. The information obtained by MDCT and findings of echocardiography were reviewed together by 2 paediatric cardiologists, and 2 cardiac radiologists with experience of more than 8 years. Perioperative anatomic descriptions, wherever available $(n=34)$, formed the gold standard for the comparison. The information obtained was classified in groups.

\section{RESULTS}

The ages of the study participants ranged 0-15 years; median 5.8 years. The male: female ratio was 0.8:1. Patient weight ranged from 2 to $55 \mathrm{~kg}$ (median $11.3 \mathrm{~kg}$ ). There were no procedure-related com- 

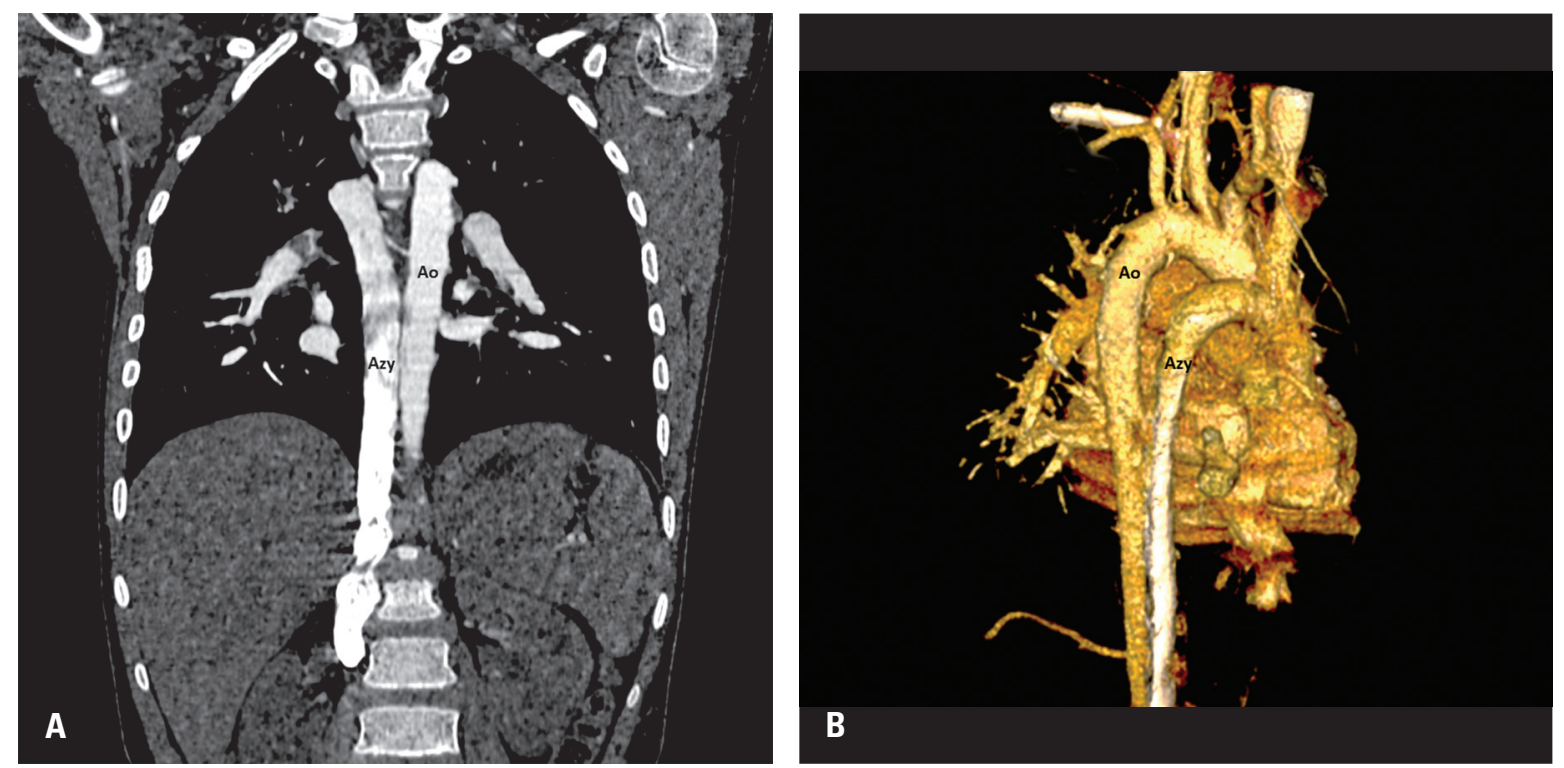

Figure 7. Coronal maximum intensity projection image $(\mathbf{A})$ and volume-rendered image $(\mathbf{B})$ showing interruption of the inferior vena cava with azygos continuation; Ao — aorta; Azy — azygos vein.
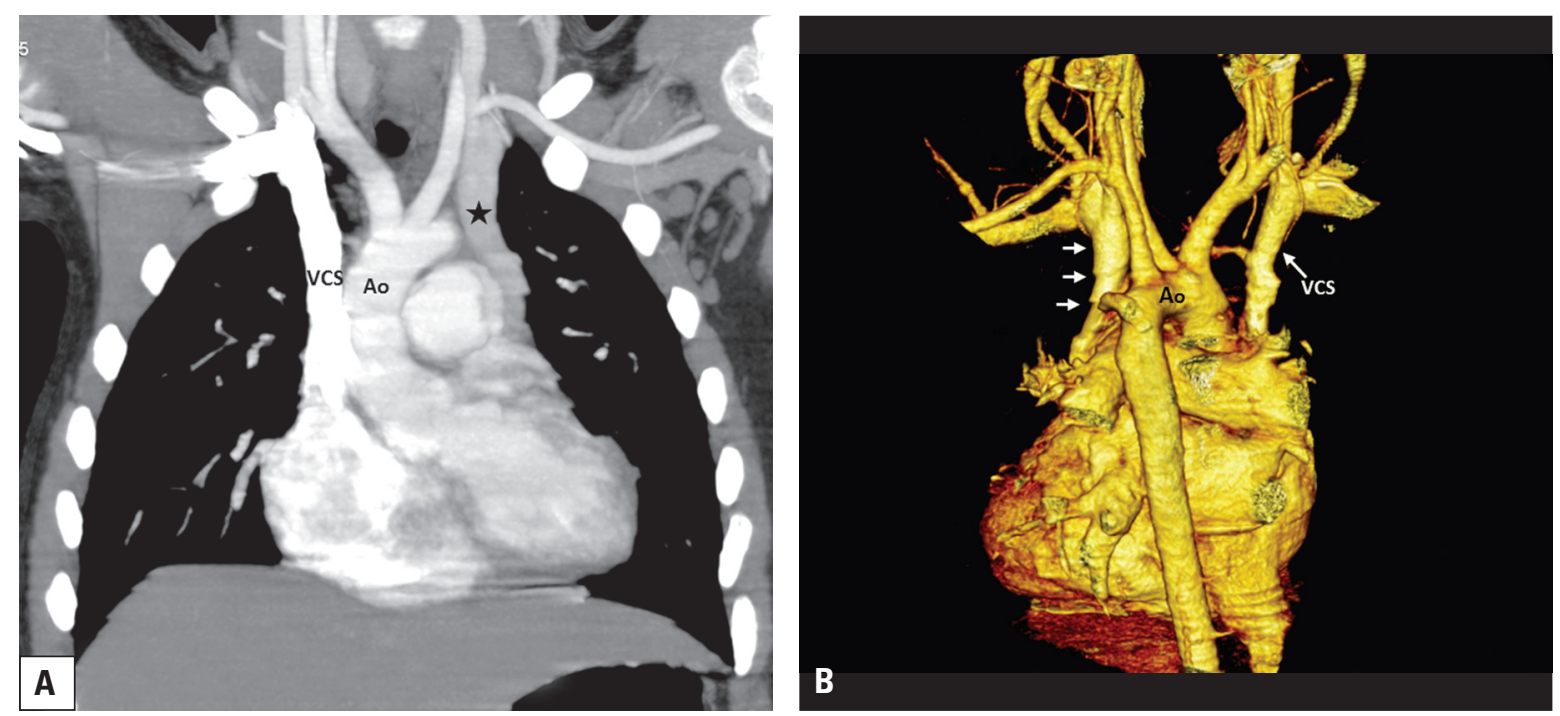

Figure 8. Coronal maximum intensity projection image (A) and volume-rendered image (B) showing persistent left superior vena cava; $A 0-$ aorta; VCS — vena cava superior; star — persistent left superior vena cava (A); arrows — persistent left superior vena cava (B).

plications. In this study the clinical consensus diagnosis defined 154 cardiovascular lesions in 102 patients and the results were compatible with available perioperative results. The results were classified in groups.

Group 1. Arterial anomalies: We found aortic coarctation in 14 patients (Fig. 1). Three patients had aortic arch hypoplasia, 3 had double aortic arch (Fig. 2), 4 had interrupted aortic arch (Fig. 3), and 2 patients had aortic pseudocoarctation. Six patients had aberrant right subclavian artery (Fig. 4); in 1 of them there was a curl in isthmus. Three patients had right aortic arch. One of them had right aortic arch with Kommerell's diverticulum (Fig. 5). Two of the patients had aortic aneurysm; 1 patient had pulmonary artery dilatation with Marfan syndrome (Fig. 6).

Group 2. Venous anomalies: We found 9 systemic vein anomalies. Two of them were interruption of the inferior vena cava with azygos continuation (Fig. 7) and 7 of them were persistent left superior vena cava (Fig. 8). We identified anomalous pulmonary venous 

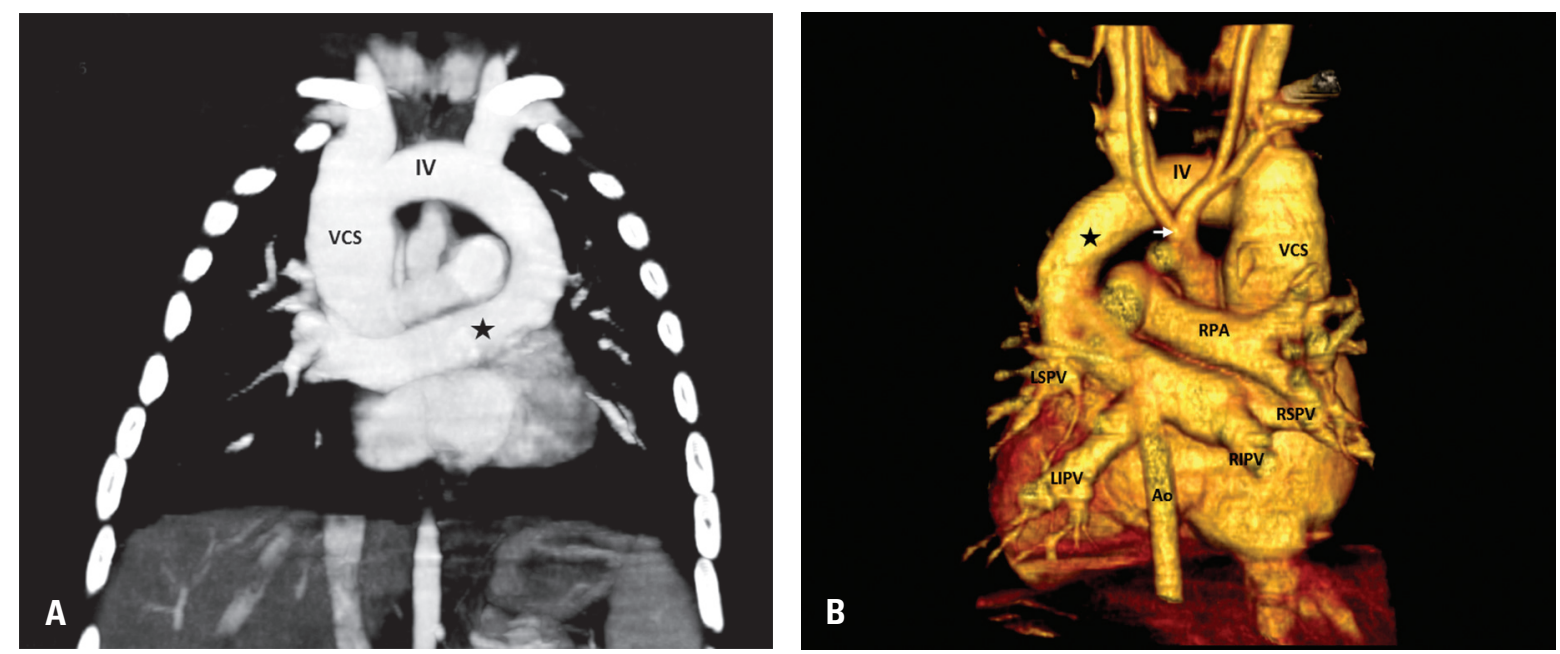

Figure 9. Coronal maximum intensity projection image (A) and volume-rendered image (B) showing drainage of pulmonary veins into the innominate vein via a common pulmonary vein in a patient with total anomalous pulmonary venous return anomaly; Ao — aorta; arrow — truncus brachiocephalicus; IV — innominate vein; RIPV — right inferior pulmonary vein; RSPV — right superior pulmonary vein; LIPV — left inferior pulmonary vein; LSPV — left superior pulmonary vein; star — common pulmonary vein; VCS — vena cava superior.
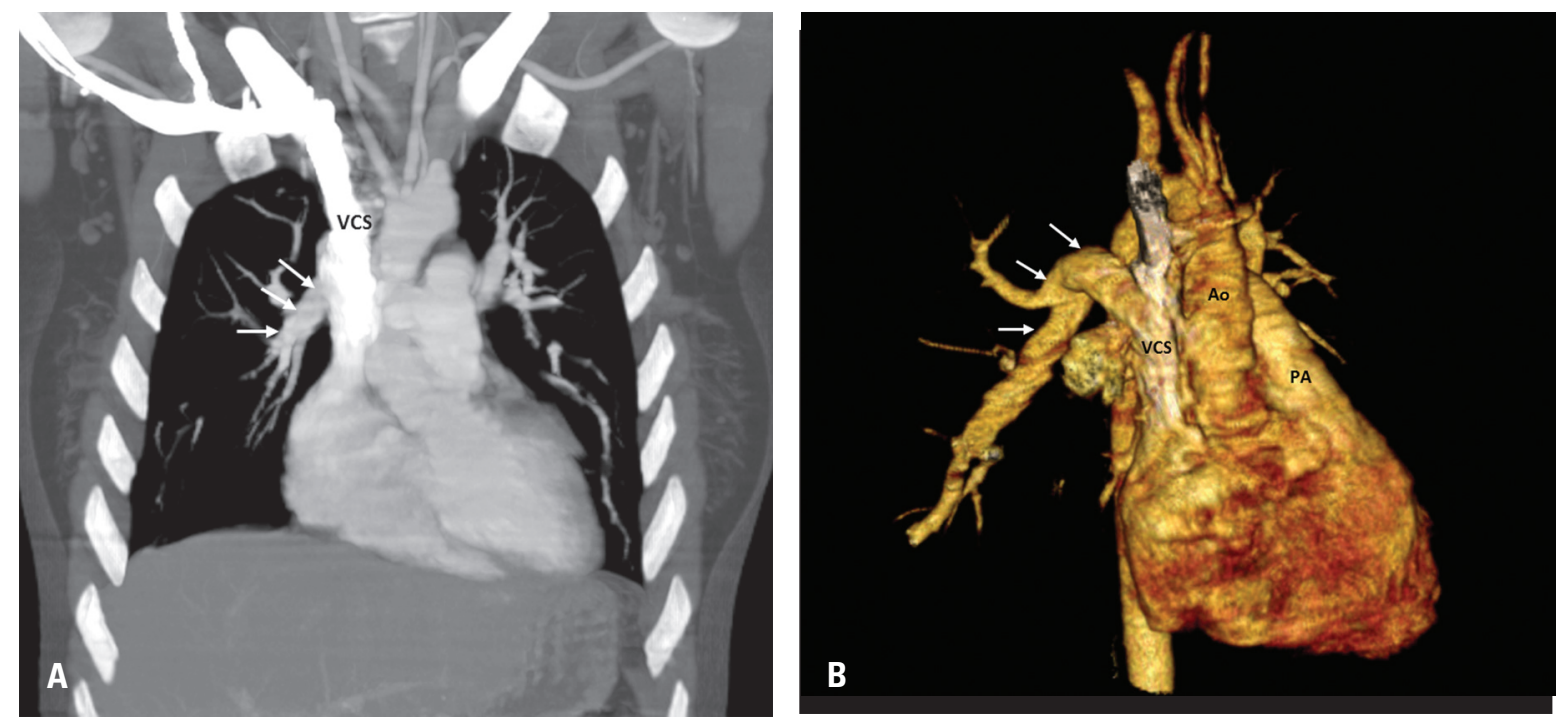

Figure 10. Coronal maximum intensity projection image (A) and volume-rendered image $(\mathbf{B})$ showing drainage of right pulmonary veins into the vena cava superior (VCS) in a patient with partial anomalous pulmonary venous return anomaly; $A_{0}$ — aorta; arrows — right pulmonary veins; PA - pulmonary artery.

return in 14 patients. Four of them were total anomalous pulmonary venous return (Fig. 9) and ten of them were partial anomalous pulmonary venous return (Fig. 10). In one of the partial anomalous pulmonary venous return there were also cardiac abnormalities such as mitral stenosis and pulmonary stenosis, patent ductus arteriosus, and atrial septal defects associated with vascular anomalies of the aorta.

Group 3. Cardiac anomalies: Seven patients had tetralogy of Fallot (Fig. 11), 8 patients had atrial septal defect, 7 patients had ventricular septal defect, and 8 patients had patent ductus arteriosus (Fig. 12). Congenitally corrected transposition of the great arteries was defined in 4 patients. In one of them the coronary arteries showed a mirror image distributions and variations (Fig. 13). Two patients had tricuspid atresia, 2 patients had cor triatriatum (Fig. 14), 2 patients had double outlet right ventricle. In 2 patients we found arrhythmogenic right ventricular dysplasia. One patient had situs inversus. 

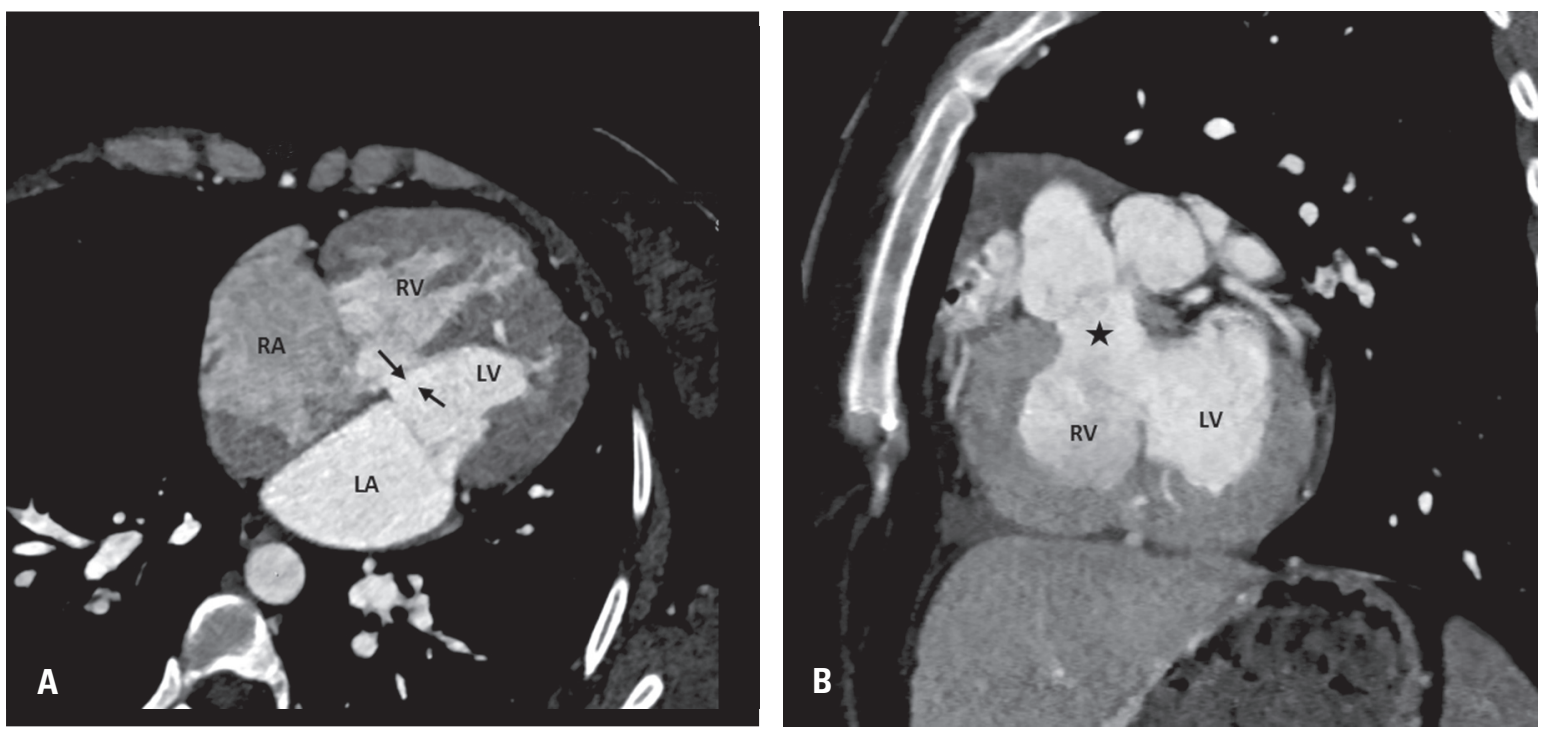

Figure 11. Axial maximum intensity projection (MIP) image (A) and sagittal oblique MIP image (B) showing ventricular septal defect (arrows) and overriding aorta in a patient with tetralogy of Fallot; LA — left atrium; LV — left ventricle; RA — right atrium; RV — right ventricle; star — overriding aorta.
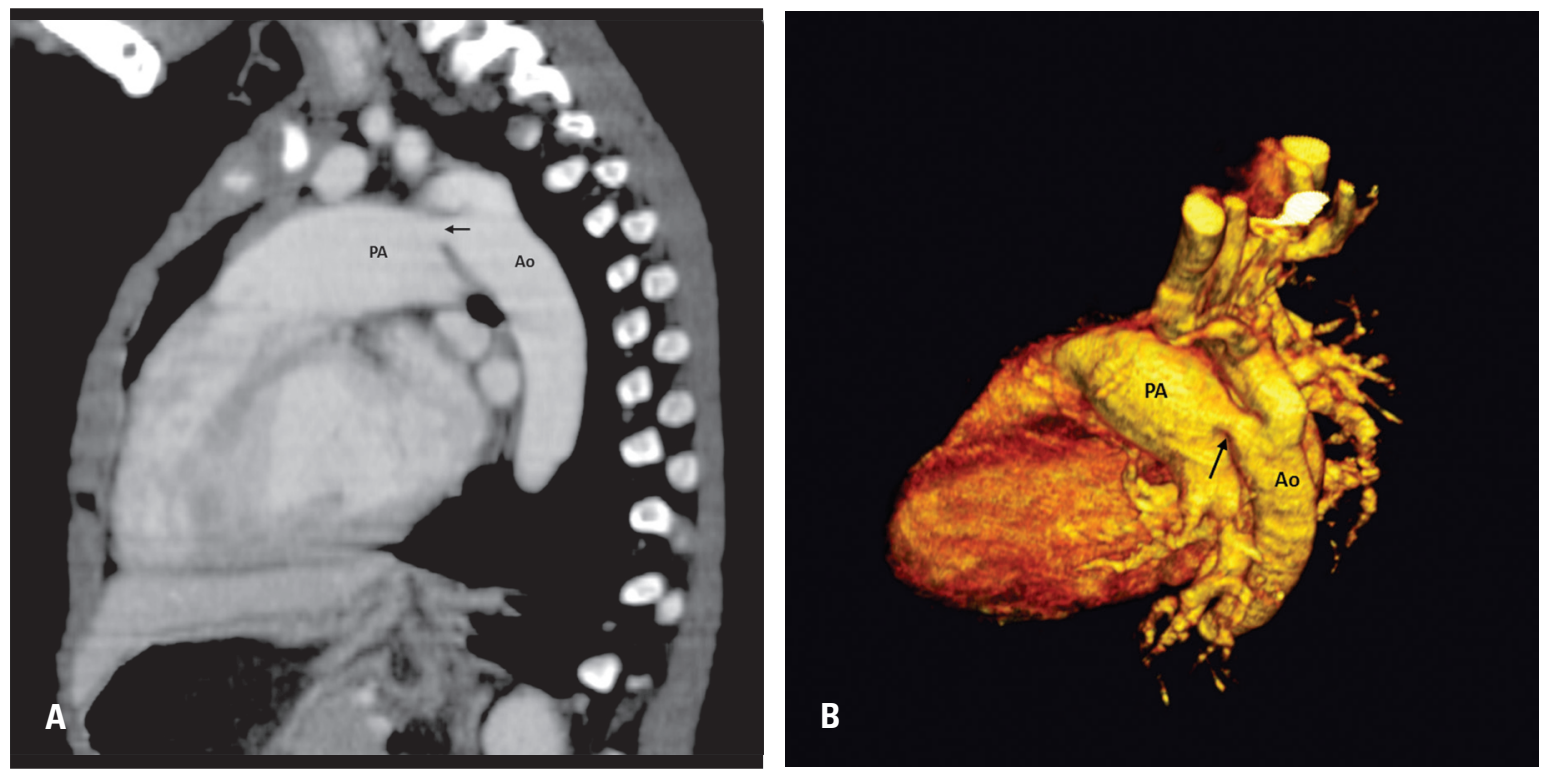

Figure 12. Sagittal maximum intensity projection image (A) and volume-rendered image (B) showing patent ductus arteriosus (arrow); Ao aorta; PA — pulmonary artery.

Beyond the cardiovascular system, MDCT found extracardiovascular lesions in $26 \%$ of the patients (26/102). Eight patients had pulmonary oedema, 11 had pneumonia, 2 had Morgagni hernia, 2 had hiatus hernia, 1 had Bochdalek hernia, 1 had congenital rib anomaly and 1 had pulmonary sequestration associated with Morgagni hernia.

\section{DISCUSSION}

CHD is a defect in the structure of the heart and great vessels which is present at birth. Heart defects are the most common birth defects and are the leading cause of birth defect-related deaths. Although echocardiography is a great modality for initial assessment due to its mobility and availability, it may not be the perfect diagnostic tool because it is usually limited by the acoustic window, spatial resolution, and the subjective interpretation of the operator [13]. Its diagnostic accuracy is only around $80 \%$ [12]. In the remaining $20 \%$ of patients, disease is hiding in the dead spaces of echocardiography, such as the pulmonary veins, aortic arch, peripheral pulmonary arteries, etc. [13]. Diagnostic catheter angiography is 

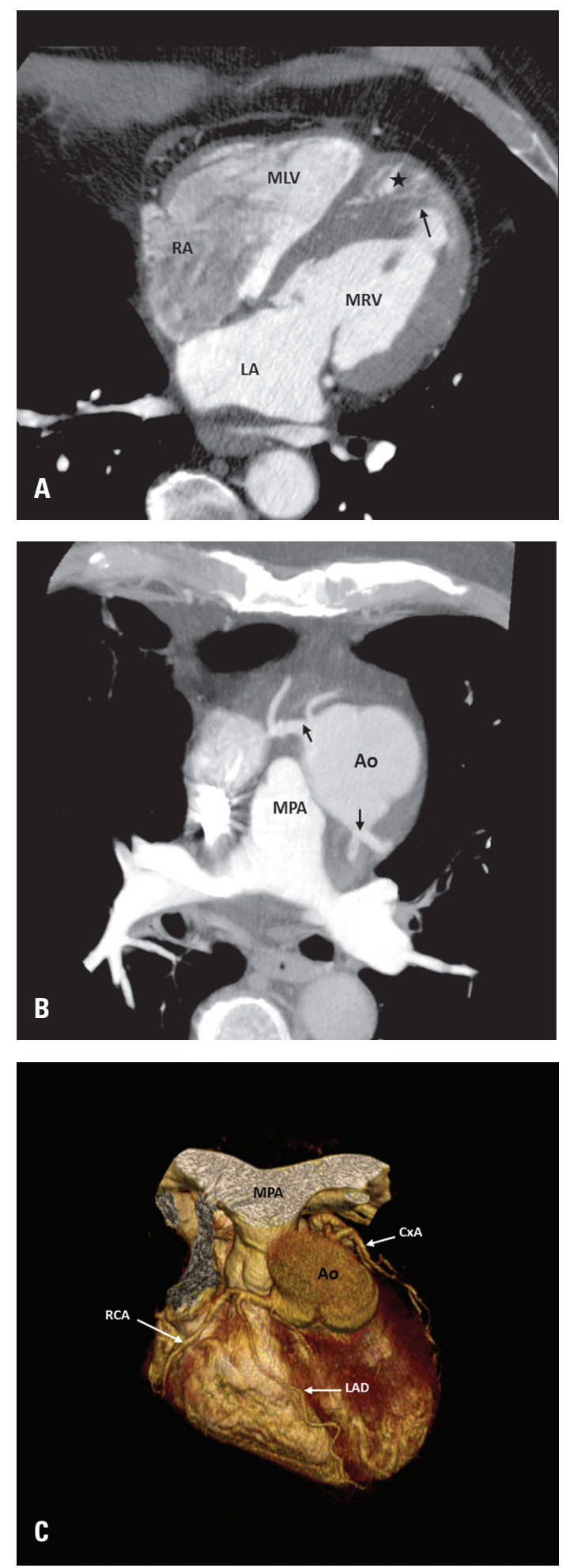

Figure 13. Axial images at the level of ventricles $(\mathbf{A})$ and aortic root (B), volume-rendered image (C) showing congenitally corrected transposition of the great arteries; A - aorta; MPA — main pulmonary artery; CXA - circumflex artery; LAD — left anterior descending artery; RCA — right coronary artery; RA — right atrium; LA — left atrium; MRV — morphologic right ventricle; MLV — morphologic left ventricle; star — trabeculation; arrow - moderator band (A); arrows — coronary arteries (B).

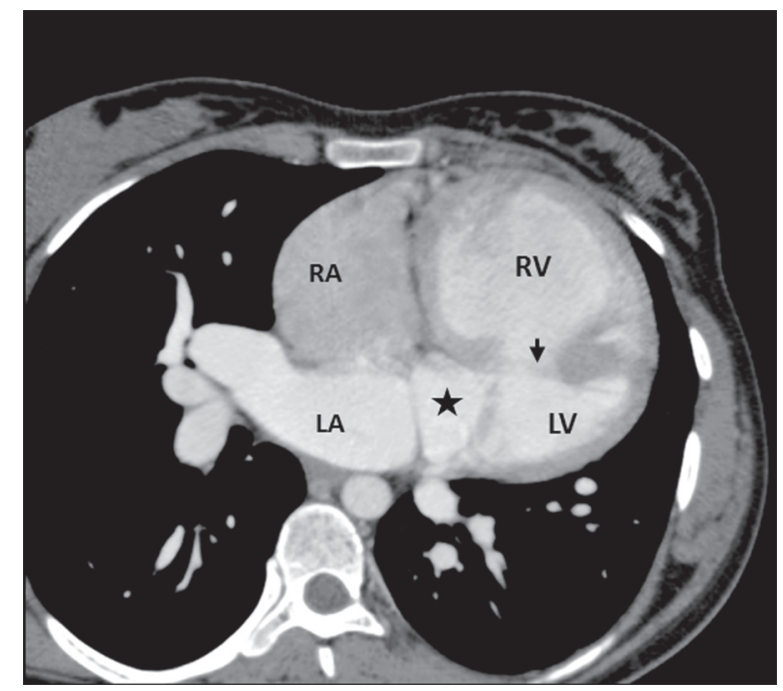

Figure 14. Axial image showing cor triatriatum sinistrum; arrow ventricular septal defect; LA — left atrium; LV — left ventricle; $\mathrm{RA}$ — right atrium; RV — right ventricle; star — third atrium.

usually recognised as the gold standard for diagnosing neonatal CHD. Catheter angiography is an invasive procedure with its inherent risks $[3,6,7]$. During this procedure, overlapping of pulmonary and systemic circulations may confuse the picture of a complex anatomy [10]. Thus, a noninvasive modality that has a higher spatial resolution and is not limited by acoustic windows is necessary. With the recent advances, MRI and MDCT now challenge the primary role of catheter angiography in many applications [13]. MRI and MDCT have an increasingly important role in the non-invasive evaluation of CHD in the pre-operative and post-operative period [11, 15]. These 2 methods may be complementary. The intracardiac anatomy is well depicted by MRI, whereas MDCT provides exquisite images of the great vessels.

MRI is time-consuming and may require patient sedation. Despite its excellent anatomic and functional assessment capabilities, high spatial resolution and fast data acquisition make MDCT an obvious modality to evaluate the unstable neonate with a small heart and complex anatomy [13]. In the situation where the clinical question is restricted to the morphology of the extra-cardiac vasculature, including the coronaries, pulmonary arteries, aorta, pulmonary or systemic veins, MDCT is comparable to MRI $[1,11]$.

MDCT takes less time, with a reduced need for sedation, is efficacious in the setting of metallic hardware, pacemakers and coils, is widely available and 
provides better definition of small vessels and higher order branches [11]. In addition, the MDCT postprocessing time is shorter than the MRI postprocessing time needed for morphologic and functional evaluation [10]. One important advantage of MDCT angiography, when compared to catheter angiography, or even MR angiography, is the ability to visualise the vessel wall [11]. MDCT also provides better delineation of the airway, mediastinal abnormalities, and the pulmonary parenchyma. Examples of indications appropriate for MDCT include, but are not limited to evaluation of total or partial anomalous pulmonary venous return, pulmonary vein stenosis, systemic and pulmonary venous anatomy in heterotaxy, branch pulmonary artery stenosis, confluence and size of branch pulmonary arteries and presence of systemic-pulmonary arterial collaterals in pulmonary atresia, vascular rings, anomalous coronaries, and the presence and severity of coarctation $[2,4,5,8,9]$. The present study showed that MDCT angiography was particularly useful in demonstrating pulmonary arteries, aortic anomalies, visceral heterotaxy and the intracardiac anatomy, systemic and pulmonary venous connection, outflow tract obstruction, and collaterals (all were well delineated). Furthermore, MDCT can simultaneously evaluate extracardiovascular lesions. In our study, $26 \%$ of the patients showed additional extracardiovascular lesions. Previous reports suggested that after initial assessment with echocardiography, MDCT could probably replace diagnostic cardiac catheterisation for further anatomic clarification for CHD in neonates [10].

The radiation is the major inherent limitation of MDCT. The most important aspect of radiation dose reduction is adjustment of the tube voltage and the current according to body weight [14]. It is important to reduce the radiation dose to as low as reasonably achievable (ALARA principle). The other disadvantage is contrast medium injection which may cause kidney burden. We have not attempted to compare CT angiography with MRI and conventional imaging techniques.

\section{CONCLUSIONS}

In conclusion, MDCT provides important information about anatomic details of CHD for the referring cardiologist. The evaluation of different anatomic structures such as heart, great vessels, lungs and abdomen is possible in one acquisition with this technique.

\section{REFERENCES}

1. Akgun V, Battal B, Karaman B, Ors F, Saglam M, Tasar M (2010) A case of anomalous left coronary artery arising from the pulmonary artery in adulthood: multidetector computed tomography coronary angiography findings. Eurasian J Med, 42: 100-102.

2. Bayraktutan U, Kantarci M, Ceviz N, Yuce I, Ogul H, Sagsoz ME, Kaya I (2013) Interrupted aortic arch associated with AP window and complex cardiac anomalies: multi detector computed tomography findings. Eurasian J Med, 45: 62-64.

3. Bayraktutan Ü, Kantarcı M, Gündoğdu F, Demirelli $S$, Yüce I, Oğul H, Duran C, Taş H, Şimşek Z, Karabulut N (2012) Efficacy of ivabradin to reduce heart rate prior to coronary CT angiography: comparison with beta-blocker. Diagn Interv Radiol, 18: 537-541.

4. Bayraktutan U, Kantarci M, Ogul H, Olgun H, Kaya I, Sagsoz ME, Ceviz N (2012) MDCT diagnosis of Infracardiac total anomalous pulmonary venous return. Eurasian J Med, 44: 188-189.

5. Bayraktutan U, Kantarci M, Olgun H, Kizrak Y, Pirimoglu B (2012) Partial anomalous pulmonary venous return associated with vascular anomalies of the aorta: multidetector computed tomography findings. Folia Morphol, 71: 115-117.

6. Goo HW, Park IS, Ko JK, Seo DM, Yun TJ, Park JJ, Yoon CH (2003) CT of congenital heart disease: normal anatomy and typical pathologic conditions. RadioGraphics, 23: 147-165.

7. Hoey E, Ganeshan A, Nader K, Randhawa K, Watkin R (2012) Cardiac neoplasms and pseudotumors: imaging findings on multidetector CT angiography. Diagn Interv Radiol, 18: 67-77.

8. Kantarci M, Koplay M, Bayraktutan U, Gundogdu F, Ceviz N (2007) Congenitally corrected transposition of the great arteries: MDCT angiography findings and interpretation of complex coronary anatomy. Int J Cardiovasc Imaging, 23: $405-410$.

9. Kantarci M, Yuce I, Yalcin A, Arslan S, Bozkurt M, Gundogdu F (2011) Evaluating adult cor triatriatum with total anomalous pulmonary venous connections by multidetector computed tomography angiography. Folia Morphol, 70: 312-314.

10. Khatri S, Varma SK, Khatri P, Kumar RS (2008) 64-slice multidetector-row computed tomographic angiography for evaluating congenital heart disease. Pediatr Cardiol, 29: 755-762.

11. Krishnamurthy R (2009) The role of MRI and CT in congenital heart disease. Pediatr Radiol, 39:196-204.

12. Soongswang J, Nana A, Laohaprasitiporn D, Durongpisitkul K, Kangkagate C, Rochanasiri W, Kovitcharoentrakul T (2000) Limitation of transthoracic echocardiography in the diagnosis of congenital heart diseases. J Med Assoc Thai, 83: 111-117.

13. Tsai IC, Chen MC, Jan SL, Wang CC, Fu YC, Lin PC, Lee T (2008) Neonatal cardiac multidetector row CT: why and how we do it. Pediatr Radiol, 38: 438-451.

14. Tsai IC, Lee T, Chen MC, Tsai WL, Lin PC, Liao WC (2007) Homogeneous enhancement in pediatric thoracic CT aortography using a novel and reproducible method: contrast-covering time. Am J Roentgenol, 188: 1131-1137.

15. Yüce I, Tanboga IH, Bayraktutan Ü, Aksakal E, Ogul H, Yalcin A, Kizrak Y, Kantarci AM (2013) Assessment of left-ventricular diastolic function in diabetic patients: the role of cardiac MR imaging. Turk J Med Sci, 43: 118-124. 